\title{
Model Policy Frameworks, Standards and Guidelines on Landslide Disaster Reduction (WCoE 2014-2017)
}

\author{
A.A. Virajh Dias, Nimesha Katuwala, H.M.J.M.K. Herath, P.V.I.P. Perera, \\ K.L.S. Sahabandu, and N. Rupasinghe
}

\begin{abstract}
The Central Engineering Consultancy Bureau (CECB) has been approved and designated as one of the "World Centres of Excellence on Landslide Disaster Reduction 2014-2017" under the theme of "Model Policy Frameworks, Standards, and Guidelines on Landslide Disaster Reduction" by the Global Promotion Committee of the International Consortium on Landslides (ICL) at the award ceremony of the World Landslide Forum 3 in Beijing, China. The above theme is divided into three thematic areas: first, Developing Conceptual Policy Frameworks to Understand the Causes, Effects and Mitigatory Measures of Landslide Occurrences, secondly to Implement Applicable Guidelines/Teaching Tools to Establish Essential Synergies in Landslide Disaster Phenomena, and thirdly to Originate Pertinent Standards for Humanitarian Activities in support of Effective Risk Reduction and Mitigations on Landslide Occurrences. The amalgamation of these three areas will originate a successive approach to developing a master plan for disaster risk reduction as a cost-effective investment in preventing future losses. The proposal for WCoE submitted by CECB was mainly focused on continuing IPL research activities, building up global partnerships and regional networks and conducting national projects and awareness programmes on Landslide Risk Reduction. This paper illustrates the above activities in a more comprehensive and descriptive manner.
\end{abstract}

\section{Keywords}

Conceptual policy frameworks - Pertinent standards - Essential synergies $・$ WCoE

A.A.V. Dias ( $\square)$

Natural Resource Management \& Laboratory Services (NRM \& LS), Central Engineering Consultancy Bureau, No. 415, Bauddhaloka MW, Colombo 7, Sri Lanka e-mail: aavirajhd@yahoo.com

\section{N. Katuwala}

Environmental Chemist, Centre for Research \& Development, NRM \& LS, Central Engineering Consultancy Bureau, No. 415, Bauddhaloka MW, Colombo 7, Sri Lanka e-mail: nkatuwala@gmail.com

H.M.J.M.K. Herath

Engineering Geologist, Center for Research \& Development, NRM \& LS, Central Engineering Consultancy Bureau, No. 415, Bauddhaloka MW, Colombo 7, Sri Lanka e-mail: jmkherath@yahoo.com

\section{P.V.I.P. Perera}

Environmental Scientist, Centre for Research \& Development, NRM \& LS, Central Engineering Consultancy Bureau,

No. 415, Bauddhaloka MW, Colombo 7, Sri Lanka e-mail: ishastha@gmail.com

K.L.S. Sahabandu Central Engineering Consultancy Bureau, No. 415, Bauddhaloka MW, Colombo 7, Sri Lanka e-mail: sahabandukls@gmail.com

\section{N. Rupasinghe}

Ministry of Megapolis and Western Development, Colombo 7, Sri Lanka

e-mail: secretarymmwd@gmail.com 


\section{Introduction}

The Central Engineering Consultancy Bureau (CECB) is one of the foremost consultancy and construction organizations in Sri Lanka today: multi-disciplinary in function and futuristic in approach towards innovative research and technology. CECB joined ICL in year 2008 with the primary objective of strengthening its research capacities and skills in Landslides and Related Earth System Disasters for Global Risk Preparedness. CECB has been an active member of the ICL/IPL-GPC since year 2008 and obtained the membership representing Sri Lanka in the ICL Capacity Development Network, which is a thematic and regional network established to support the regional activities of ICL and IPL. CECB has also been admitted as a Member of the Board Representatives under the status and bylaws of ICL in all matters dealing with landslide initiatives in year 2012. In year 2014, CECB became one of the 15 World Centres of Excellence (WCoE) in Landslide Disaster Reduction for the three consecutive years; 2014-2017 under the title of "Developing Model Policy Frameworks, Standards and Guidelines on Landslide Disaster Reduction".

This paper systematically describes the completed and on-going IPL research components and WCoE activities conducted by CECB for capacity building and knowledge dissemination purposes on Landslide Disaster Reduction.

\section{ICL/IPL Activities of CECB}

- The institution has been a member of the ICL/IPL-GPC since 2008 and is a member of the ICL Capacity Development Network.

- CECB has also been admitted as a Member of the Board of Representatives of ICL in all matters dealing with landslide initiatives since 2008.

- CECB participated in the First World Landslide Forum (WLF1) which was held in Japan, 2008 and also represented Sri Lanka from CECB in the WLF2 held in Rome, Italy in 2011 and WLF3 held in Beijing, China in 2014.

- Participated and presented papers at the IPL Symposiums/chairing ICL-GPC sessions.

- Published five peer-reviewed papers based on diversified experiences obtained in the field of landslide disaster phenomena for the WLF3 held in China, 2014.

- Elected as a World Centre of Excellence in Landslide Disaster Reduction for the period 2014-2017 at the WLF3 held in Beijing, China.

- Conducted the International E-conference under the theme of Developing Model Policy Frameworks, Standards and Guidelines in Landslide Disaster Risk Reduction as an initiative for the WCoE activities steered by CECB.

\section{National Projects}

As a partner of the IPL-GPC, our institution was strengthened to support many of the national level programmes based on landslide disaster management. We contributed our active participation as a member of the Disaster Impact Assessment checklist systems for the Road Development Sector of the Disaster Management Capacity Enhancement Project (DiMPEP), organized by JICA and DiMPEP. Our expertise was utilized towards the national-level Landslide Disaster Mitigation Action Plan, "Operational Professional Combine" during landslides and floods that occurred in Sri Lanka in 2003.

\section{ICL/IPL Research Projects}

During the recent past, CECB has conducted ICL-driven research projects with a problem/solution focus, strengthened by the research team of the Centre for Research \& Development Unit of CECB. The project details can be elaborated as follows.

\section{IPL Certified Project M143-Year 2009}

Evaluation of Sensitivity of the Combined Hydrological Model (Dynamic) for Landslide Susceptibility Risk Mapping in Sri Lanka.; Team Leader-Eng. A. A. Virajh Dias

This study includes the quantification of the landslide hazard in terms of spatial and temporal occurrences of slope stability in a sub catchment, based on a combined ground water and slope stability dynamic model for the selected area. A comparative analysis of landslide susceptibility was done using the WAA and SINMAP static models (see map in Fig. 1) and using the PC Raster Combined Hydrological Dynamic Model (see map in Fig. 2). The landslide susceptibility maps created from both the models were compared and used for a better understanding of landslide susceptibility for sensitive landslide disaster events, their origins and prioritization of efforts for the reduction and mitigation of future landslide hazards. The sensitivity of both the approaches was fine-tuned using soil strength parameters, geomorphological evidence and field verification techniques.

The above findings indicate the variability of conceptual models and their relative importance with respect to the input databases. Detailed and comprehensive geographical information databases are required to obtain the statistical interpretation and calibration of the model. Similarly, soil saturation conditions and the validity of wetness indices within a watershed also create another avenue to calibrate the analytical model with the inputs of other soil parameters (Dias et al. 2014a). 


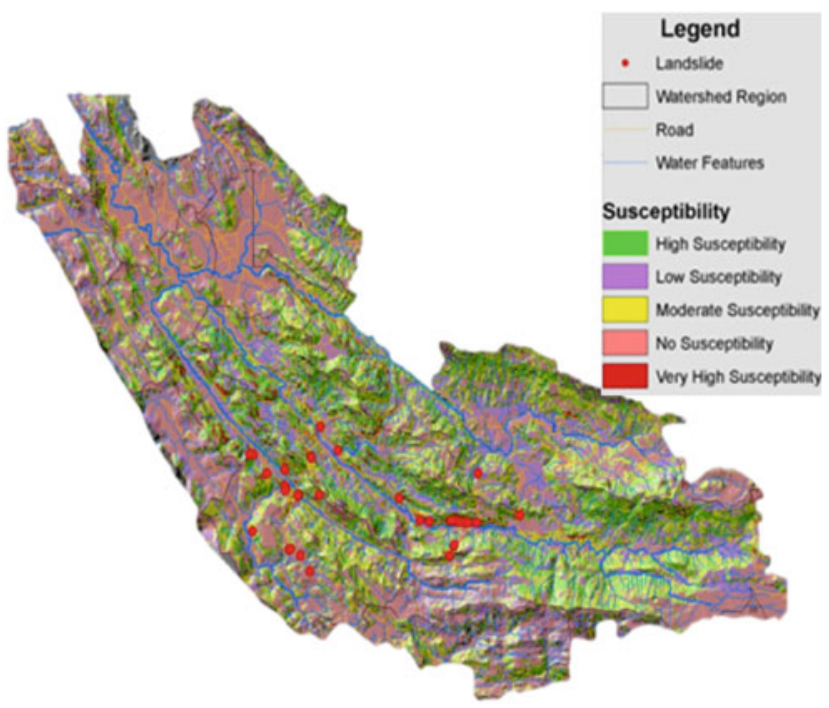

Fig. 1 Detailed landslide susceptibility map of Kalawana division of the Ratnapura District, developed using the SINMAP model, including stream hydrology networks, roads and watershed boundaries

Fig. 2 Landslide susceptibility of a selected area interpreted through combined hydrological dynamic model (PC Raster). Blue to Red indicates increases in hazard potential due to continuous rainfall and saturation within a period of 6 days

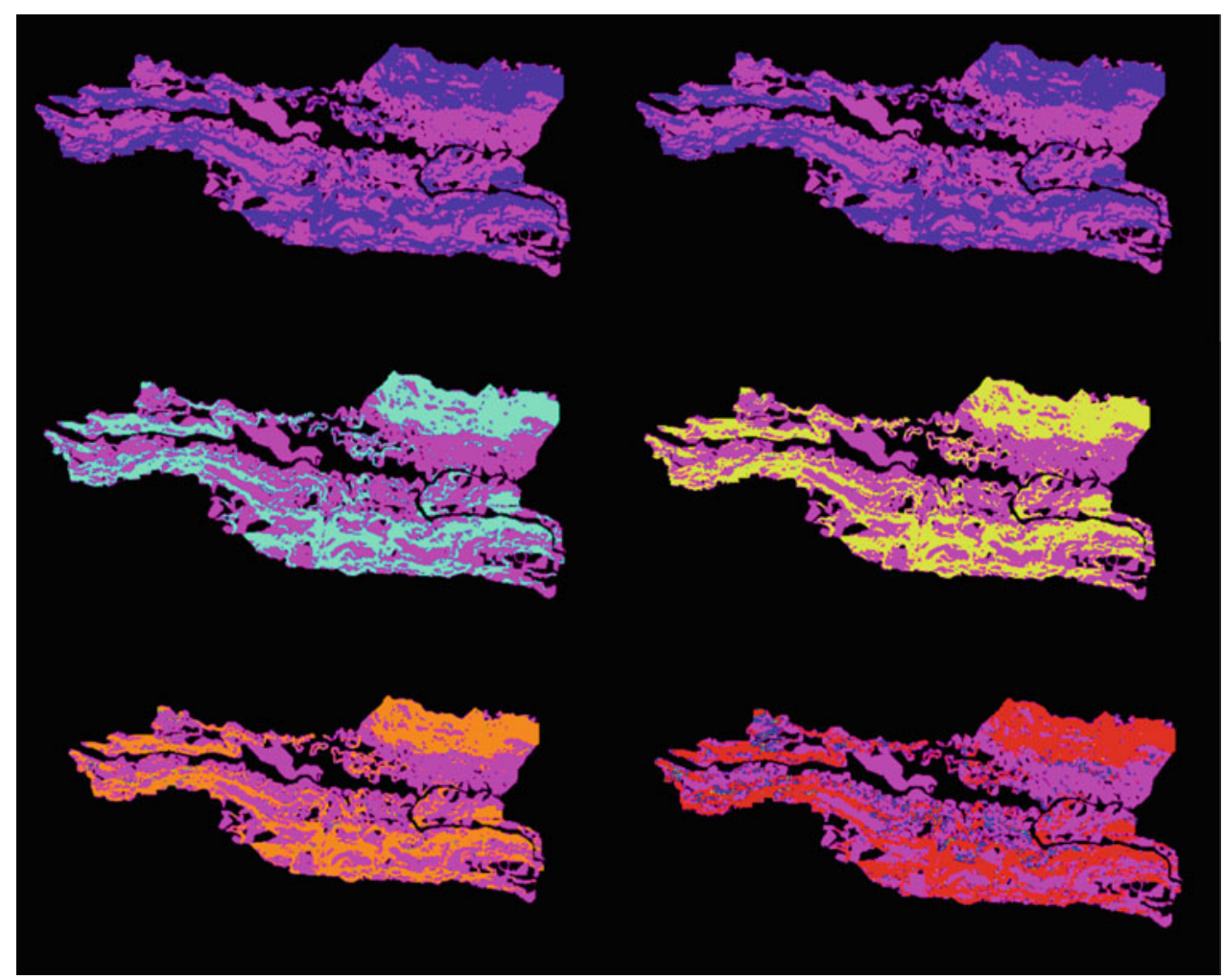

\section{IPL Certified Project 155-Year 2012}

Determination of Soil Parameters of Subsurface to be used in Slope Stability Analysis in two Different Precipitations Zones of Sri Lanka; Team Leader-Eng. A. A. Virajh Dias

The characteristics of slopes, saturation and shear strength of soils are the main parameters associated with rainfall-induced slope failures, and these parameters are directly affected by differences in precipitation over prolong periods of time. In most instances a landslide is triggered due to extensive soil saturation and is a function of soil integrity, hydraulic conductivity, density, void content, shear strength and boundary conditions (Dias et al. 2014b). The study on evaluation of E50 (Secant modules) is an experiment set up to understand the behavior of residual soils under changing 




Fig. 3 Relationship of effective confining pressure and soil moldule $\mathrm{E}_{50}$

stress conditions at a site due to various reasons, such as prolong periods of rainfall, movement of soils, unloading effects and the re-loading effect caused by deposition. From the results we conclude a reasonable interdependence of effective (confining) pressure and E50. One of the difficulties faced during the experimental study was to apply the evaluation of degree of landslide density considering a special boundary (area wise) to the selected sample, when comparing prior to evaluation of parametric results which could have influenced the final results, as shown in Fig. 3 (Mallawarachchi et al. 2014).

\section{IPL Certified Project 199—Year 2015}

The Effect of Root Systems in Natural Slope Erosion Protection in the Hill Country of Sri Lanka.- on going project; Team Leader-Mr. Ishastha Perera

This study reports the observed details and patterns of vegetation which support slope protection and the roles played by different species in such scenarios. It is understood that not one, but a collection of species contribute to this end through the setting and functions of each type of vegetation and their positioning.

Thus results of the study can be directly used for practical application to critical slopes. For example, the large single root usually which grows straight down, anchors the plant in the ground and the connected lateral roots anchor the soil, preventing soil erosion. The buttress root system, which is distributed on all sides of a shallowly rooted tree, does not

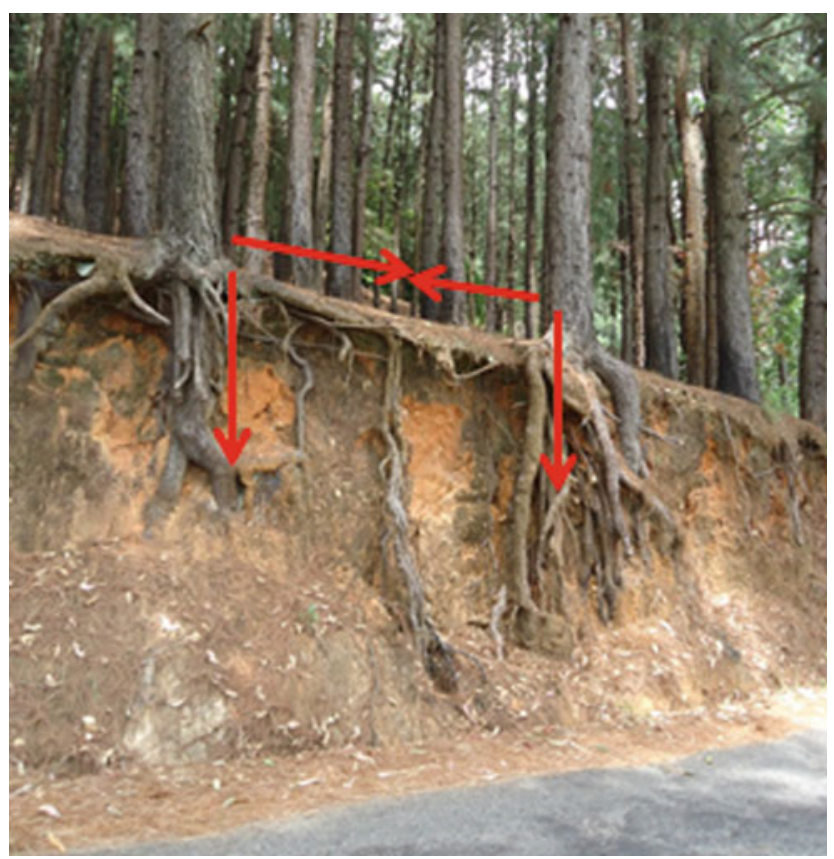

Fig. 4 The large single root (taproot) and the lateral roots connected to anchor the soil, prevent soil erosion

penetrate to deeper surface layers (see Fig. 4). It prevents the tree from falling over, while also gathering more nutrients.

\section{IPL Certified Project 200—Year 2015}

An assessment of the rock fall susceptibility based on cut slopes adjacent to highways and railways - on going project; Team Leader-Ms. H M Janaki M K Herath

The main target of this research is to carry out appropriate improvements for rock fall hazard assessment by introducing an appropriate Rockfall Hazard Rating System (RHRS). This method indicates various judgment matrices, but it does not clearly define a method of assigning individual weights by prioritizing their significance. Roadside overhanging rock slope failures are commonly due to removal of a passive loading wedge, dipping, continuous joints with adverse orientation, direct exposure to heating or freezing, undulating joint sets, differential erosion, layered strata, disparate rock block sizes, geo-structural discontinuities and the slope.

\section{Approach to Rating Using Geological Evidence}

Determining the rating scores for pair-wise geological characters is more convenient than individual geological significance of RHRS. The rating and scoring of evidence still depend on value judgments, but avoids the minimum dependences of other factorial significance and pairwise combined parameters. The study discusses some prominent 


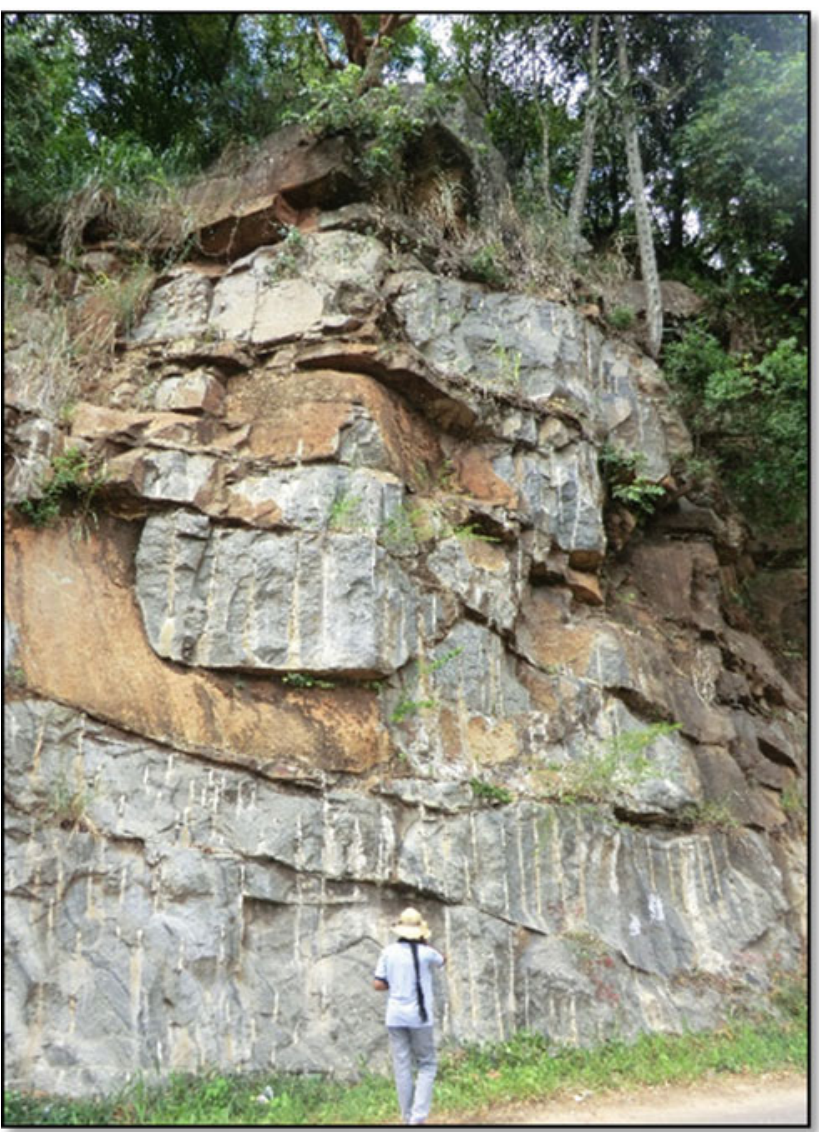

Fig. 5 This relatively old cut slope indicates progressive erosion, with unsupported or over-steepened slope conditions and degradation over time. The detachment of larger blocks or volumes of falling rock produces greater impact forces than smaller events. This type of rockfall is commonly caused by erosion that leads to a loss of support, either locally or throughout the slope

characteristics in geological settings, which include time-dependent parameters of rock fall history obtained from the field evidence, similar to that shown in Fig. 5.

The descriptive information related to the study is given below.

\section{Pair 1: Dipping and Slope Height (DSH)}

The instability of a cut slope is directly interrelated with the height of the earth cutting. A consideration of the combined parameters of dipping and slope height will demarcate the potential instability more precisely than independent evaluation. Therefore, DSH can be considered as the first of the combined parameters for the evaluation of RHRS.

\section{Pair 2: Block Size and Rock Character (BRC)}

It is noticable that different rocks disintegrate to form various sizes of blocks, depending on climatic conditions, weathering, etc. Therefore, the formation of blocks largely depends on the rock character or lithology. Crystalline gneissic rocks with large blocks, well foliated gneissic rocks and bedded/fractured quartzite are considered to be examples in that category.

\section{Pair 3: Persistence and Joint Density (PJD)}

Continuous planar joints, discontinuous joints and random joints were common in most of the cases. Random joints and fractures create irregular shaped blocks. Therefore, the persistence and joint density affect detachment of blocks from a cutting. All comparisons were to be made in the vertical or horizontal axis. Planar joints and foliation joints are prominent observations and joint density is essential to defining the potential instability.

\section{Pair 4: Weathering Discontinuity and Aperture Sizes (DAS)}

In general, formation of rock blocks is a function of the discontinuities of the intact rock. The potential for rockfall by movement along discontinuities is controlled by the characteristic of joints or the aperture size. The condition of the joints is described in terms of micro and macro roughness. The aperture size always indicates the possibility of absorbing water and saturation potential. In addition, water ingress through discontinuities always reduces the strength of an interface and increase weathering. Therefore the nature of the discontinuity (fracture, joint, fault) of the mass of rock and observations of aperture size is an important combined parameter for the assessment.

\section{Pair 5: Rock Friction and Weathering State (RFWS)}

The angle of internal friction of intact rock is usually high in crystalline formations. However, the interface friction of the facture or joint surface will indicate the state of stability. Quartzite and charnockitic formations tend to remain unweathered. Rock friction is a function along a joint, bedding plane, or other discontinuity which is governed by the macro and micro roughness of the surfaces. Macro roughness is the degree of undulation of the joint relative to the direction of possible movement in a rock mass. Micro roughness is the texture of the surface, which is mostly based on the weathering of the slope.

\section{Pair 6: Cutting Plane and Block Movement Direction (CPBMD)}

Cutting plane is the exposed face of the cutting which may have an adverse effect on the orientation of a detachment of blocks. The study of cutting faces with respect to the block orientation is used for slopes where, for differential blocks, over-steepening and under-steeping conditions may control or aggravate the possible detachment of overhanging rocks. 


\section{Pair 7: Overhang and Fall History (ODH)}

Historical rockfalls and the detachment of adjoining blocks at a rock cut is an indicator of future instability events. Typically, the frequency and magnitude of past events is an excellent indicator of the type of events to expect. In addition, overhanging rocks are mainly due to the fall history and therefore this information is an important check on the potential for future failure. The second stage of a rockfall event is when the large overhang becomes unstable and a large volume of rock falls off at one time. Erosion features include over-steepened slopes, unsupported rock units (overhangs), or exposed resistant rocks on a slope, which may eventually lead to a rockfall event. Therefore overhang of rocks with fall history is a useful combined parameter for the revised RHRS (Herath et al. 2014, 2016).

\section{WCoE Activities Conducted by CECB}

\section{Developing "Model Policy Frameworks, Standards and Guidelines on Landslide Disaster Reduction"}

The Central Engineering Consultancy Bureau was designated as a "World Centre of Excellence on Landslide Disaster Reduction 2014-2017" under the theme of "Model Policy Frameworks, Standards, and Guidelines on Landslide Disaster Reduction" by the Global Promotion Committee of the International Consortium of Landslides (ICL) at the World Landslide Forum 3 held in Beijing, China.

A conceptual model policy framework is a tool with numerous variations and contexts which are used to make conceptual distinctions and organize ideas towards the focus area. The development of a Policy Framework can be done for diverse goals, including the development of a Policy Framework to promote and enrich landslide risk awareness, as well as to promote a multi-disciplinary culture of landslide studies and management, developing policies to breed and nurse scientific culture and outlook, etc. Once the Policy Framework is clearly defined for a particular task, the applicable standards and guidelines can be implemented accordingly.

\section{The International E-Conference 2015 on Landslide Disaster Risk Reduction}

CECB has taken the initiative in organizing an Online Global Conference on Landslide Disaster Reduction, finding it an ideal way of providing ample room for respective specialists to assemble the world-wide knowledge and expertise in disaster risk management to better serve and care for the needs of society.
The primary objective of conducting this E-conference is to develop a Master Plan for Landslide Risk Mitigation in the world by identifying the critical factors that may directly affect it and develop a set of Teaching Tools/Guidelines which are more practical and suitable to the level of understanding of the actual victims. The conference preceded under the theme of Developing Model Policy Frameworks, Standards \& Guidelines on Landslide Disaster Reduction, which was derived from the theme for work as a WCoE by CECB. It was divided into three subordinate topics; the opening topic was Developing Conceptual Policy Frameworks to Understand the Causes, Effects and Mitigatory Measures of Landslide Occurrences, secondly was the Implementation of Applicable Guidelines/Teaching Tools to Establish Essential Synergies in Landslide Disaster Phenomena, and thirdly to Originate Pertinent Standards for Humanitarian Activities in support of Effective Risk Reduction and Mitigations on Landslide Occurrences. Several important factors discussed throughout the conference are described below (Bhandari 2015).

\section{Are landslide hazards natural?}

Hazards due to landslides, earthquakes, volcanoes and tsunamis are generally regarded as nature's safety valves which operate to maintain Earth's dynamic equilibrium. It is a hard fact of life that mountains of the world, which are treasure-houses of landslides, are meant by Nature to decay and without their degradation; slope failures and mass movements, there would be no fertile plains for agriculture. Therefore, Nature maintains its dynamic equilibrium by getting rid of human interferences with the mountain system, such as urbanization, illegal constructions, inappropriate land use patterns in cultivation, etc. The sharp increase in tragic landslide events is mostly because of this reason. It is certainly unfair to regard landslides as natural hazards when such massive dominating non-natural causative factors have been added, changing the very character of hazard The importance of an accepted policy framework in land-use planning comes into picture at this stage.

2. How sound are the approaches to landslide risk reduction and mitigation?

Projection of landslide scenarios and estimation of the associated risk are pre-requisites for formulating strategies to reduce or eliminate landslide risk. Risk is defined as "the combination of the probability of an event and its negative consequences". Hazard is defined as "a dangerous phenomenon, human activity or condition that may cause loss of life, injury or other health impacts, property damage, loss of livelihoods and services, social and economic disruption, or 
environmental damage". It can be calculated risk as the product of hazard and vulnerability.

Within the landslide risk management framework, vulnerability is regarded as potential for damage and loss caused by a hazard and would depend on a random mix of physical, social, economic, and environmental factors, which interact in space and time.

3. Development of a Policy Framework to promote and enrich the landslide risk awareness

The landslide hazard map is another important entity in developing a policy framework to enrich landslide risk awareness. It is essential that the policy context be field validated, reliability certified and user-friendly. It should be effectively utilized, ensuring timely mitigation and expedited response. A special policy context should be outlined for the use of architects, planners, engineers and contractors to certify the safety of structures they plan, design and build. It will be useful to provide these hazard maps and policies to foreign visitors to educate them about the hazards they might face. If an area has been identified as vulnerable to landslides and categorized under the landslide-prone areas, awareness programmes should be immediately conducted for the local community. This should be handled with utmost care, since the local community has been established in these areas making use of the local resources and involved in regional occupations. Thus it is important to build an amicable and lasting relationship with them. The state authorities and intervening organizations should consult these communities on proposing alternative job opportunities and highlighting the benefit of resettling in a safer environment to minimize the impact in catastrophic situations. It is of vital importance to build a strong rapport with these citizens and thereby counteract or avoid misconceptions and negative attitudes towards the policies and action plans proposed by the government (Rupasinghe et al. 2014).

\section{Development of a Policy Framework to promote a multi-disciplinary culture of landslide studies and management}

The major factor that governs promoting the multi-disciplinary culture of landslide studies is none other than the landslide professionals. It is essential to create a gradational structure, spreading from a large well branched network which acts as a tremendous supportive system of sharing information and services among individuals and groups until it reaches the grass-roots level. This network can be utilized in conducting effective awareness programmes, educating each and every individual in the network on how to avoid, minimize and mitigate the impacts of catastrophic phenomena. Thus every individual should build up their own moral strength. when acting in areas of landslide occurrence, with a sensible approach of leaving such localities and settling down in safer surroundings. This moral strength should be built up bit by bit, until they identify with avoiding vulnerability, including cultivation in slope areas, establishing domestic environs in mountainous regions and occupying landslide-prone regions for employment. Such attempts can only succeed through a greater interaction between professionals pursuing landslide studies and those considering other types of hazards, in pursuit of the common cause of advancement of knowledge and human safety.

5. Do the landslide risk reduction policies breed and nurse a scientific culture and outlook?

Once the landslide risk reduction policy framework has been structured it is worthwhile to analyze whether these policies breed and nurse a scientific culture and outlook. In most developing countries, there is a near absence of policies promoting scientific outlooks in landslide studies. This may be due to the shortfall of resources and inadequate capacities available in third-world countries to manage landslides. Yet there is an urgent requirement to set up policies that drive the culture of scientific scrutiny before allowing investments in landslide disaster risk reduction. All aspects in mitigation strategies should be taken into account to develop deeper insights initially. It is more supportive if this hydra-headed social problem is disassembled into smaller fragments, first considering one at a time and then ultimately as a cluster. For instance, when considering a scientific investigation of a post-landslide occurrence, the most preliminary fact to establish is whether the investigation team is multi-disciplinary in expertise and skilled in exercising engineering judgment to do justice to the landslide study at the moment.

6. How focused are we on what we need to ensure landslide risk reduction?

Classifications based on diagnostics are required by the landslide specialist, whereas decision makers and disaster managers would prefer risk-based classification. Risk is related to damage or losses caused by the occurrence of hazards, both in the short term and in the long term. While in the grip of a disaster, the primary concerns are anticipated losses due to deaths and injuries. Further it could be aimed at 
persons who are untraceable, damage to buildings, infrastructure, and goods and services. Concerns such as post-disaster lootings, social unrest, environmental degradation, loss of agriculture will also emerge. However the consequences of the phenomena are not easy to quantify. A few of the intangible losses include halting of the process of development caused by disruption and dislocation.

\section{Early warning against landslides}

Landslides are among those geohazards which are predictable. Yet it generally costs a lot to utilize possible early warning systems against landslides. Thus a third-world country cannot solely depend on its government to establish such facilities. It is essential that the communities are empowered to promote simple and economical instrumentation and trained to foresee the danger of landslides for themselves. People are impressed by the magical power of modern technology, but for the developing world, it would be inappropriate to over-look the advantages of ease, economy, accessibility and time-tested benefits of traditional technologies. It is also time to think in terms of community-centric early warning systems.

8. Viewing landslide studies in the larger context of disaster risk reduction

Landslide risk can no longer be considered in isolation. Other hazardous events such as floods and earthquakes are also entangled with landslides to a certain extent. It can be further introduced as landslide-triggered tsunamis, earthquake-induced landslides, and flood-induced landslides. Since a landslide-prone area could also be flood- and earthquake-prone, it is only by the production of multi-hazard maps that the scientists can connect the universe of landslides with the galaxy of other disasters, and encourage a universal approach to hazard investigation and disaster risk reduction.

9. What do we intend to leave for posterity by way of documentation on diverse and kaleidoscopic aspects of landslides?

The vast rage of research papers and diversified study reports naturally reflect more of the perceptions of the writers, built up on a pile of observations and past experience, and well known for loyalty to the widely accepted trends and theories.

The research teams should be encouraged to re-visit scientifically controversial, but educationally important case-records, not just with a view to either verify or falsify whatever has been concluded earlier, but to let the truth surface naturally through the true spirit of scientific enquiry. Modern technology allows us to repeatedly test the lessons learned and use those very lessons to perform differently. It is depressing to observe the growing disconnect between landslide investigation and landslide remediation, and the gap seems to be widening because of the commercially-driven recourse to a brutal use of technology to fix problematic slopes and landslides.

Finally, Conceptual Model Policy Frameworks will be considered successful if landslide risk reduction becomes a way of life in the national development agenda and breeds the culture of safety in day-to-day lives.

\section{Summary of Statistics of the International E-Conference 2015}

The E-conference was an amalgamation of 300 participants representing almost all the continents in the world and 20 keynote experts/facilitators, supporting an effective communication platform throughout the project (see Figs. 6, 7 and 8). The E-conference was carried out through an official website (http://e-conference.crdcecbsl.1k/) specifically created as an open source medium to enable all viewers to connect simultaneously to share their ideas and comments related to the topic.

A Local Forum was also run in parallel with the International E-conference in order to confer in depth on the pressing matters due to landslides of national significance. A consultation workshop was conducted to identify the thematic areas to come up with feasible solutions for the issues faced by vulnerable communities from the grass-roots level. A Question Session was also instigated in order to clarify all the issues that have emerged throughout the conference and utilized the expertise knowledge in finding the best solution for these issues.

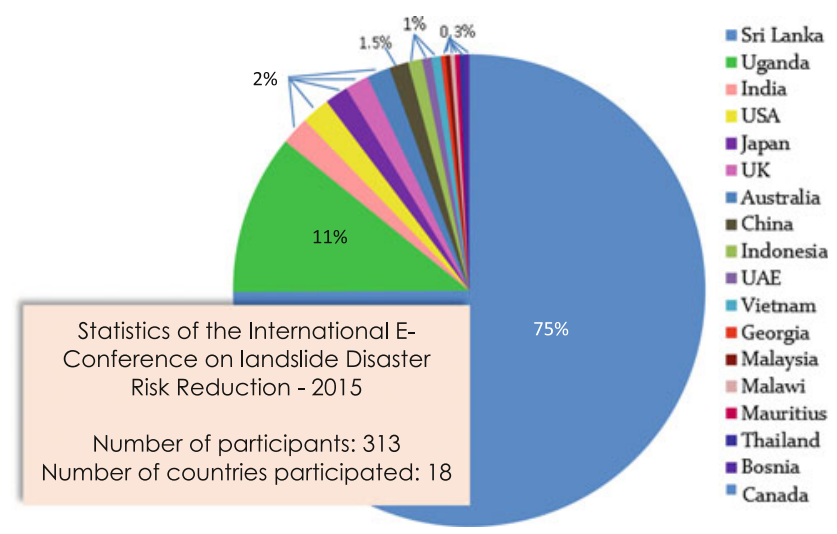

Fig. 6 Percentage of participants by country 


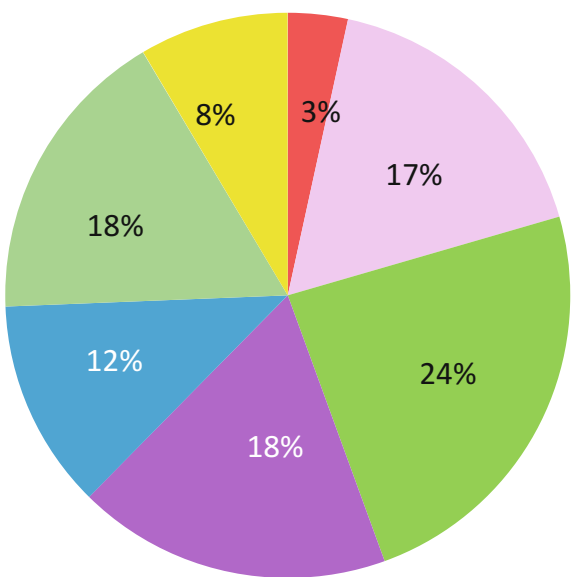

- UN Organizations

International Org.(related to Disaster Mgt.)

n Governmental Organizations

- Social/Community based Organizations

academic -Local

In Academic- Foreign

Other

Fig. 7 Percentage of participants by affiliation

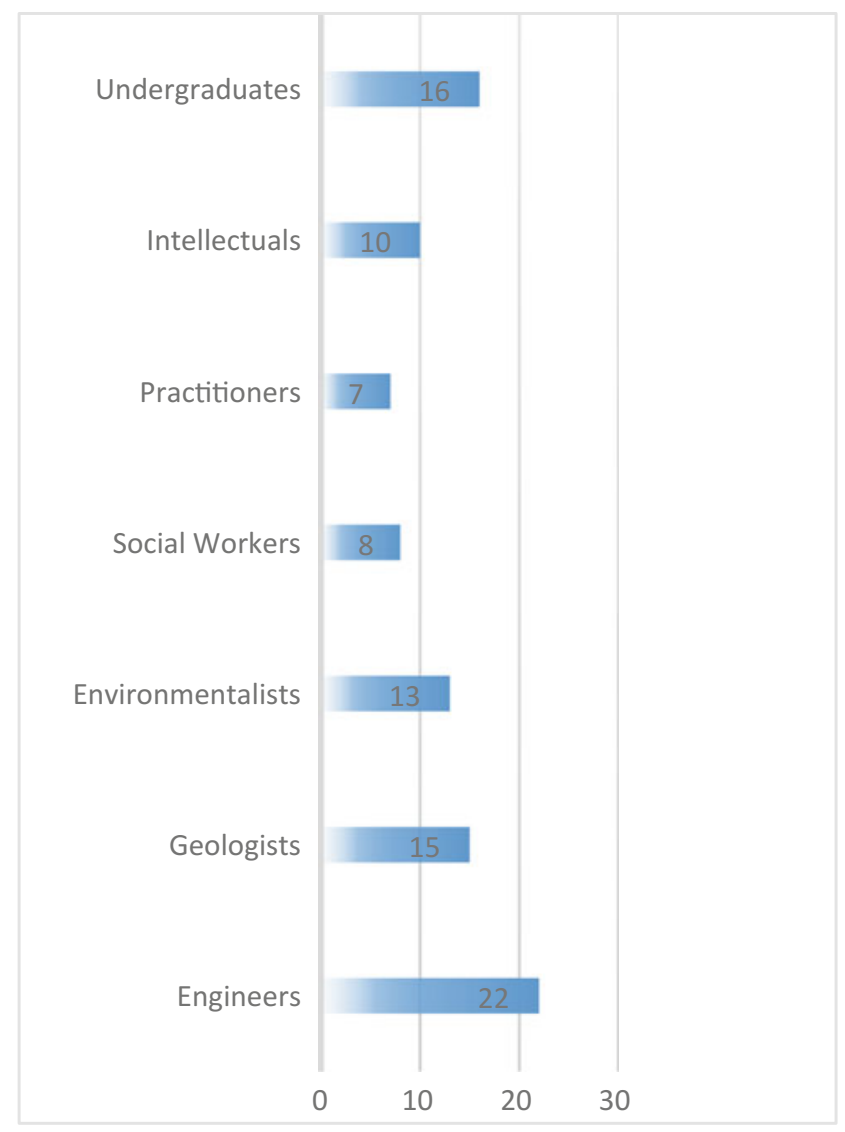

Fig. 8 Percentage of participants by career
The experts reviewed the globally available necessary information, identified research needs and gaps in existing recommendations directly relevant to the respective problems. The summary of the key areas discussed will be published in the conference proceedings.

The main objective of publishing the proceedings is to filter the most significant facts discussed throughout the conference in order to increase the readability and obtaining the maximum usage of it by the society. The institutional target is focused more towards the social aspects, such as enriching landslide risk awareness among citizens (including school students, workmen and laymen) and ensuring the effective transmission of this message to the public to enhance their adaptability and familiarity with the mechanisms involving landslide mitigation and slope protection measures discussed throughout the conference. It is our pleasure to work in partnership with UNDP towards this approach. It was certainly a fruitful global discussion in support of bringing a sustainable humanitarian future to fruition.

Acknowledgements This paper deals with integral parts of the research projects conducted under the International Programme on Landslides: IPL-143, IPL-155, IPL-199 and IPL-200, which have been implemented by the Centre for Research \& Development, Natural Resources Management \& Laboratory Services, and the Central Engineering Consultancy Bureau (CECB). The CECB is the premier consultancy organisation in Sri Lanka, with a wide range of expertise in different engineering disciplines of the Ministry of Mahaweli Development \& Environment. It is published with their permission. The views expressed in the paper are however those of the authors only. Our grateful thanks are due to the Eng. G. D.A. Piyathilaka, Chairman, and Eng. K.L.S. Sahabandu, General Manager, CECB for permission and encouragement.

\section{References}

Bhandari RK (2015) Developing conceptual model policy frameworks to understand causes, effects and mitigations of landslide occurrences. In: International E-conference on landslide disaster reduction-2015, Sri Lanka 24th March-24th May 2015

Dias AAV, Gunathilaka AAJK (2014a) Evaluation of sensitivity of the WAA and SINMAP models for landslide susceptibility risk mapping in Sri Lanka. In: Proceeding of the World Landslide Forum3 (WLF3), Beijing, China, 2-6 June 2014, vol 2, Landslide Science for a Safer Geoenvironment, pp 167-173

Dias AAV, Abayakoon SBS, Bhandari RK (2014b) Discrete boundary shear strength of a landslide at high rainfall precipitation zone in Sri Lanka. In: Proceeding of the World Landslide Forum3 (WLF3), Beijing, China, 2-6 June 2014, vol 1, Landslide Science for a Safer Geoenvironment, pp 101-106

Herath HM, Janaki MK, Kodagoda SSI, Dias AAV (2014) Shallow modes of slope failure in road earth cuttings in Sri Lanka. In: Proceeding of the World Landslide Forum3 (WLF3), Beijing, China, 2-6 June 2014, vol 2, Landslide Science for a Safer Geoenvironment, pp 51-58

Herath HM, Janaki MK, Jayasooriya JADNA, Dias AAV (2016) Geological stability of overhanging rock slopes. In: Proceedings of 2016 IPL Symposium, UNESCO, Paris, November 17-18, 2016 
Mallawarachchi MASN, Ekanayake EMTM, Kodagoda SSI, Dias AAV (2014) Comparison of soil modulus E50 of residual soil slope failures in two different rainfall zones. In: Proceeding of the World Landslide Forum3 (WLF3), Beijing, China, 2-6 June 2014, vol 1, Landslide Science for a Safer Geoenvironment, pp 135-141
Rupasinghe N, Dias AAV, Hennayake SK (2014) Role of intervening agencies and officials in emergency risk management of landslides, Sri Lanka. In: Proceeding of the World Landslide Forum 3 (WLF3), Beijing, China, 2-6 June 2014, vol 4, pp 700-705
Open Access This chapter is licensed under the terms of the Creative Commons Attribution 4.0 International License (http:// creativecommons.org/licenses/by/4.0/), which permits use, sharing, adaptation, distribution and reproduction in any medium or format, as long as you give appropriate credit to the original author(s) and the source, provide a link to the Creative Commons license and indicate if changes were made.
The images or other third party material in this chapter are included in the chapter's Creative Commons license, unless indicated otherwise in a credit line to the material. If material is not included in the chapter's Creative Commons license and your intended use is not permitted by statutory regulation or exceeds the permitted use, you will need to obtain permission directly from the copyright holder. 